\title{
Technical note: Monte Carlo genetic algorithm (MCGA) for model analysis of multiphase chemical kinetics to determine transport and reaction rate coefficients using multiple experimental data sets
}

\author{
Thomas Berkemeier ${ }^{1,2}$, Markus Ammann ${ }^{3}$, Ulrich K. Krieger ${ }^{4}$, Thomas Peter $^{4}$, Peter Spichtinger $^{5}$, Ulrich Pöschl ${ }^{1}$, \\ Manabu Shiraiwa ${ }^{1,6}$, and Andrew J. Huisman ${ }^{7}$ \\ ${ }^{1}$ Multiphase Chemistry Department, Max Planck Institute for Chemistry, 55128 Mainz, Germany \\ ${ }^{2}$ School of Chemical and Biomolecular Engineering, Georgia Institute of Technology, 30320, Atlanta, GA, USA \\ ${ }^{3}$ Laboratory of Environmental Chemistry, Paul Scherrer Institute, 5232 Villigen, Switzerland \\ ${ }^{4}$ Institute for Atmospheric and Climate Science, ETH Zurich, 8092 Zurich, Switzerland \\ ${ }^{5}$ Institute for Atmospheric Physics, Johannes Gutenberg University, 55128 Mainz, Germany \\ ${ }^{6}$ Department of Chemistry, University of California, Irvine, 92697, Irvine, CA, USA \\ ${ }^{7}$ Department of Chemistry, Union College, 12308, Schenectady, NY, USA
}

Correspondence to: Thomas Berkemeier (thomas.berkemeier@chbe.gatech.edu)

and Andrew J. Huisman (huismana@union.edu)

Received: 18 January 2017 - Discussion started: 23 January 2017

Revised: 9 May 2017 - Accepted: 21 May 2017 - Published: 30 June 2017

\begin{abstract}
We present a Monte Carlo genetic algorithm (MCGA) for efficient, automated, and unbiased global optimization of model input parameters by simultaneous fitting to multiple experimental data sets. The algorithm was developed to address the inverse modelling problems associated with fitting large sets of model input parameters encountered in state-of-the-art kinetic models for heterogeneous and multiphase atmospheric chemistry. The MCGA approach utilizes a sequence of optimization methods to find and characterize the solution of an optimization problem. It addresses an issue inherent to complex models whose extensive input parameter sets may not be uniquely determined from limited input data. Such ambiguity in the derived parameter values can be reliably detected using this new set of tools, allowing users to design experiments that should be particularly useful for constraining model parameters. We show that the MCGA has been used successfully to constrain parameters such as chemical reaction rate coefficients, diffusion coefficients, and Henry's law solubility coefficients in kinetic models of gas uptake and chemical transformation of aerosol particles as well as multiphase chemistry at the atmosphere-biosphere interface. While this study focuses on the processes outlined above, the MCGA approach should be portable to any nu-
\end{abstract}

merical process model with similar computational expense and extent of the fitting parameter space.

\section{Introduction}

Atmospheric aerosols play a key role in climate, air quality, and public health. Heterogeneous reactions and multiphase processes alter the physical and chemical properties of organic aerosol particles, but the effects of these reactions are not fully elucidated (e.g. Finlayson-Pitts, 2009; George and Abbatt, 2010; Abbatt et al., 2012; Pöschl and Shiraiwa, 2015). While multiphase chemistry in aerosols and clouds can be described by a sequence of well-understood physical and chemical elementary processes in kinetic models (Hanson et al., 1994; Pöschl et al., 2007; George and Abbatt, 2010), the deduction of parameters or rate coefficients of the individual elementary processes is severely complicated by the inherent coupling of chemical reactions and mass transport processes (Kolb et al., 2010; Berkemeier et al., 2013; Shiraiwa et al., 2014).

Heterogeneous chemical reactions on aerosol particles are traditionally described using so-called resistor models, which 
represent parallel and sequential physical or chemical processes in analogy to electrical circuits. These models have typically been used to derive analytical expressions for simplified limiting cases (e.g. Hanson et al., 1994; Worsnop et al., 2002; Hearn et al., 2005). Recently, numerical models have been developed that allow a more complete consideration of the time- and depth-resolved chemical and physical behaviour of aerosol particles, leading to a better understanding of these reaction systems, especially under conditions where the steady-state assumptions underlying the resistor models are not valid (Smith et al., 2003; Pöschl et al., 2007; Steimer et al., 2015; Berkemeier et al., 2016). Kinetic multilayer models describe single particles or thin films by division into compartments such as near-surface gas phase, surface, and particle bulk and further subdivision of the particle bulk into thin layers to achieve depth resolution. Specific models provide a focus on chemistry (KM-SUB, Shiraiwa et al., 2010), on gas-particle partitioning (KM-GAP, Shiraiwa et al., 2012, and ADCHAM, Roldin et al., 2014), or on water diffusion (the ETH Diffusion Model, Zobrist et al., 2011). For simplicity, throughout this paper we refer to a kinetic model as any computational model that is used to simulate a system's behaviour. We will use the term input parameters to address the prescribed model parameters (thermodynamic, kinetic, or physical) that are optimized in this study so that kinetic model output matches experimental data, a process that we will refer to as fitting the kinetic model. Note that this definition excludes model parameters that are clearly defined by physical laws or the experiment (e.g. physical constants and experimental conditions) or are of purely technical nature (e.g. integration time steps).

Ideally, fitting a kinetic model to experimental data would return all chemical and physical parameters necessary to understand the importance of the processes at work and to predict the outcome of future experiments, even if conducted under experimental conditions not part of the training data set, i.e. all experimental data used during the fitting process. However, kinetic models often require a multitude of input parameters, some of which are not constrained well experimentally or are merely effective parameters combining a sequence of inherently coupled processes. In general, two main difficulties arise when optimizing complex models to experimental data:

1. The optimization hypersurface is often non-convex, i.e. it will not have only a single minimum due to interactions between non-orthogonal (coupled) input parameters and/or scatter in the experimental data. Hence, steepest descent methods fail since they get trapped easily in local minima. Brute-force or exhaustive searches, where an $n$-dimensional grid is applied to the input parameter space and the fit quality evaluated for every grid point in all $n$ dimensions, are often not computationally feasible.
2. If too few or too similar experimental data are used during the fitting process or input parameters are allowed to move in a large range, the optimization problem can be underdetermined (ill-defined) and multiple solutions may exist. In this case, even though a good agreement between model output and training data set is obtained, it is likely that only the model input parameters corresponding to the most limiting processes will be physically meaningful. Extrapolation of the model outside its training range can then lead to strong discrepancies between modelled and measured data. For example, if a model is trained using data that are exclusively limited by a single process, it will constrain the parameters that represent that specific process while the other parameters remain nearly unconstrained even if multiple data sets are used. This means that if a parameter set were optimized using data from surface film experiments, the bulk diffusion coefficients would likely be poorly constrained regardless of how many different experimental data sets of that type were used.

Hence, sophisticated optimization methods, which quickly and reliably determine the model input parameters that lead to the best correlation between kinetic model and experiment, are needed. Furthermore, experiments covering a broad range of conditions must be conducted to ensure that the observables are controlled by (a) as many model input parameters as possible across all experimental conditions, but (b) by as few model input parameters as possible for a specific experimental condition (i.e. limiting cases). The MCGA presented here is able to overcome the difficulty of a complex optimization hypersurface with many local minima while providing the user with a realistic assessment of how well-constrained the model input parameters are by the experimental data.

\section{Monte Carlo genetic algorithm (MCGA)}

In many modelling applications, methods are needed that reliably find the optimum in non-convex optimization problems and detect underdetermined optimization problems. Global optimization methods have been subject of extensive research in the past (Arora et al., 1995) and provide means of approximating non-convex optimization problems without premature convergence to local optima. Examples for these methods are simulated annealing methods and evolutionary algorithms. In atmospheric chemistry, simple optimization techniques are commonly used to determine kinetic parameters by fitting rate equations to experimental data sets. However, to our knowledge no global optimization technique diligently designed for the determination of atmospheric reaction rate coefficients from multiple data sets has been described thus far. A related technique (Markov chain Monte Carlo algorithm) has been used to determine parametric uncertainties in cloud-aerosol interaction models (Partridge et 


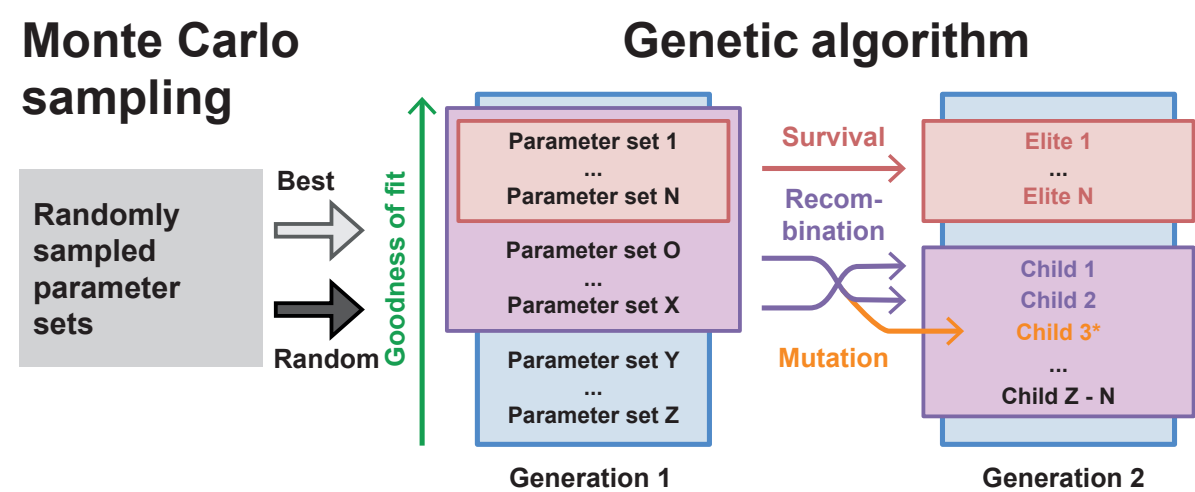

Figure 1. Schematic representation of the MCGA optimization method consisting of a Monte Carlo sampling, which feeds into a genetic algorithm. Populations of model input parameter sets (blue boxes) are iteratively improved over several generations through survival of elites (red boxes) and recombination and mutation of parents to create children (purple boxes) until a sufficient correlation to the experimental data (goodness of fit) is obtained.

al., 2012; Lowe et al., 2016). Global optimization was also used to calculate thermodynamic equilibria for phase separation of aqueous multicomponent solutions (Zuend and Seinfeld, 2013).

In this study, we present the Monte Carlo genetic algorithm (MCGA), a method combining direct Monte Carlo sampling with a genetic algorithm as a heuristic global optimization method that approximates the global optimum for input parameter sets of computational models. Repeated execution of the search algorithm can be used to test for uniqueness or to provide statistical bounds on the model input parameters. The MCGA utilizes a two-step approach to find minima on non-convex hypersurfaces. First, a Monte Carlo (MC) sampling is performed in the large space of possible model input parameters to narrow down the possible solution to smaller areas of interest. The parameter sets are evaluated using a goodness-of-fit expression of the user's choice, such as the RMSE between kinetic model output and experimental data. In the examples presented here, the RMSE or logarithmic RMSE was used. When multiple data sets were fitted, a weighting factor was introduced to prevent bias due to the number of data points in different experimental data sets. An additional optional weighting factor allows the user to assign priority to experimental data with lower statistical error or scatter. The parameter sets for the MC sampling are generated randomly from a distribution of the model input parameters. Each parameter was sampled using a logarithmically spaced distribution of values to provide uniform sampling over the large ranges most input parameters can possibly adopt. Note that, depending on the problem, different distributions and sampling strategies (e.g. Latin hypercube sampling) could be applied.

The genetic algorithm (GA) uses survival of the fittest to optimize an ensemble (the population) of parameter sets (the individuals) over several iterations (the generations). Processes known from natural evolution such as survival, recombination, mutation, and migration are mimicked to optimize a population. The initial population is formed by the parameter sets with the best goodness of fit obtained in the MC sampling step. An equal number of random parameter sets are added to ensure diversity within the pool of parameter sets and counteract sampling bias from shallow local minima (Fig. 1).

During execution of the GA, a number of model input parameter sets with the highest correlation between model output and experimental data (goodness of fit) are directly transferred into the next generation by the survival mechanism (the elites). The remaining population is generated using combinations of parameters from the individuals in the previous generation with moderate or better goodness of fit (the parents), forming the children for the next generation. In this study, $5 \%$ of the next generation are elite individuals, which are transferred with no changes, while $80 \%$ of the children are created by randomly choosing individual parameters (genes) from two selected parents with equal weighting. The higher the goodness of fit of a certain individual, the higher its likeliness to be selected as a parent. This way, parameters leading to high goodness of fit are positively reinforced, leading to improvement and slow homogenization of the population. Finally, $20 \%$ of children are created by applying a mutation scheme that alters parameters in a stochastic manner within the prescribed bounds to enhance genetic variability. Collectively, these mechanisms enable the MCGA to overcome local minima, a crucial feature of a global optimization method. Iteration of these steps eventually results in a homogeneous, optimized population and the common parameter set is taken as a result. The MCGA can be run multiple times to generate a set of representative solutions, which has been the default approach in previous applications of MCGA (see Sect. 4). With only a few ( 510) repetitions, this procedure allows the user to assure full convergence to the global optimum. In addition, the random sampling of optimization space between different executions of MCGA will generate statistical bounds on the parameters 


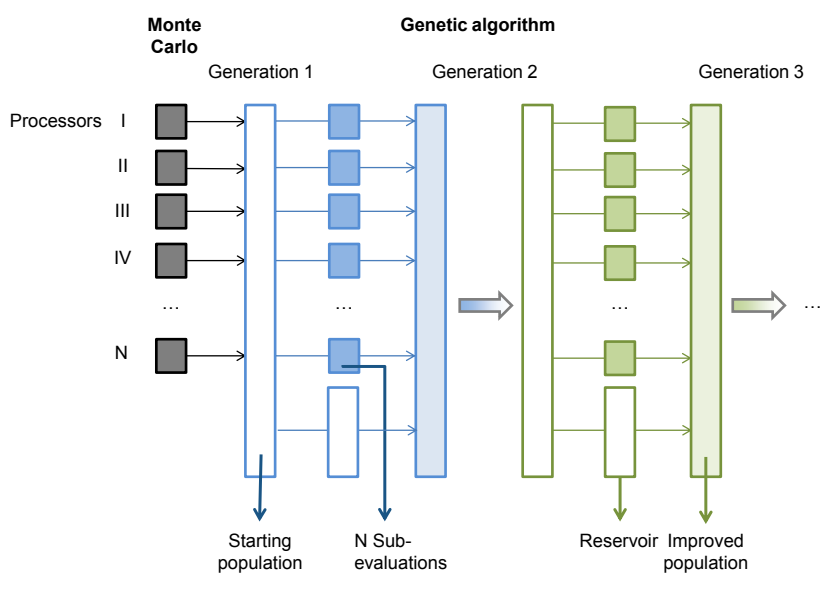

Figure 2. Schematic visualization of the parallelized MCGA optimization method. The Monte Carlo step is performed independently on $N$ processors and the best-fitting parameter sets are fed along with random parameter sets into the starting population. During the genetic algorithm step, each processor extracts a number of parameter sets from the collective pool and performs a sub-evaluation of the genetic algorithm on these parameter sets. After completion, the optimized parameter sets are fed back into the pool, which always contains a non-zero number of parameter sets as a reservoir. After randomization, a different combination of parameter sets is extracted and the process is repeated.

if a sufficiently large number of repetitions is computationally feasible.

In this study we used the genetic algorithm provided by MathWorks ${ }^{\circledR}$ (MATLAB ${ }^{\circledR}$ Global Optimization Toolbox) and developed a routine for parallel computation on computer clusters. In a typical setting, the MC step and GA step of the optimization occupied an approximately equal amount of computation time. Figure 2 describes the implementation of the parallel MCGA optimization method. The $N$ parallel threads share common populations of parameter sets that are iteratively optimized by extracting a subset of parameter sets and performing the genetic algorithm on this subset. Once a sub-evaluation of the genetic algorithm has finished, the parameter sets are mixed into the population, and after randomization, a different subset of parameter sets is extracted and their optimization is immediately continued. Since the parallel threads will run asynchronously, a fraction of individuals must remain in the population to be mixed to enable continuous operation without waiting times.

\section{Implications for modelling and measuring chemical kinetics}

Although models may possess a multitude of kinetic and thermodynamic input parameters that represent the many possible sequential and/or concurrent processes occurring in the system, their behaviour is often driven by only a single or at most a few processes at a certain point in time. In chemical kinetics, the behaviour of the system can often be characterized by a kinetic regime, which may change during the course of the reaction and with experimental conditions (Berkemeier et al., 2013). If a set of model input parameters can be uniquely determined (by MCGA or other means) and results in a high-fidelity fit of model output to experimental data, the parameters would then be regarded as correct within the approximations of the underlying model and uncertainties of the experimental data. This is a convenient way to assimilate data from multiple previous studies; data sets can be weighted to reflect confidence in their results, and the final range of accepted parameters then represents a consensus from the fitted data. However, it may not always be possible to fully constrain the input parameters, even using multiple experimental data sets. In general, there are two reasons that a model input parameter can remain unconstrained after optimization.

i. The parameter is non-influential.

ii. The parameter is inherently coupled to another one, forming a non-orthogonal parameter pair under all experimental conditions.

Figure 3 illustrates both cases in an example taken from atmospheric multiphase chemistry, using the benchmark system of ozone plus oleic acid and data adopted from Hearn et al. (2005). The original data were converted from ozone exposure to a time series using an ozone concentration of $2.76 \times 10^{15} \mathrm{~cm}^{-3}$. The MCGA was executed under a constrained parameter set in which only desorption lifetime and surface reaction rate coefficient were allowed to vary. In this scenario, repeated execution of MCGA returned multiple solutions, for which the model output had nearly equivalent goodness of fit with only slight variance between them (Fig. 3a). In stark contrast to the uniform correlation between model output and experimental data, Fig. 3b shows the high variance within the model parameters yielding these solutions (red markers), which scatter across a narrow valley of the optimization hypersurface (contour lines). In the upper portion of the figure, i.e. at desorption lifetimes larger than $10^{-4} \mathrm{~s}$, a vertical relationship between both parameters indicates that the desorption lifetime is a non-influential parameter and can take on any value in this interval, corresponding to case (i) above. In the lower portion of the figure, i.e. below a desorption lifetime of the diagonal relationship indicates that an increase in one parameter can be compensated with a decrease in the other parameter and both form a non-orthogonal pair, corresponding to case (ii) above. For comparison, Fig. 3c and d show examples of optimization hypersurfaces from Berkemeier et al. (2016), who studied multiphase ozonolysis of shikimic acid and investigated the existence of non-orthogonal parameter pairs by varying optimized parameters $\left(\lambda_{i}\right)$ by a factor $f\left(\lambda_{i}\right)$ to depict the total residual as a 2-D contour map. Figure $3 \mathrm{c}$ shows that the Henry's law coefficient for ozone $\left(\mathrm{H}_{\mathrm{cp}, \mathrm{O}_{3}}\right)$ and the product 

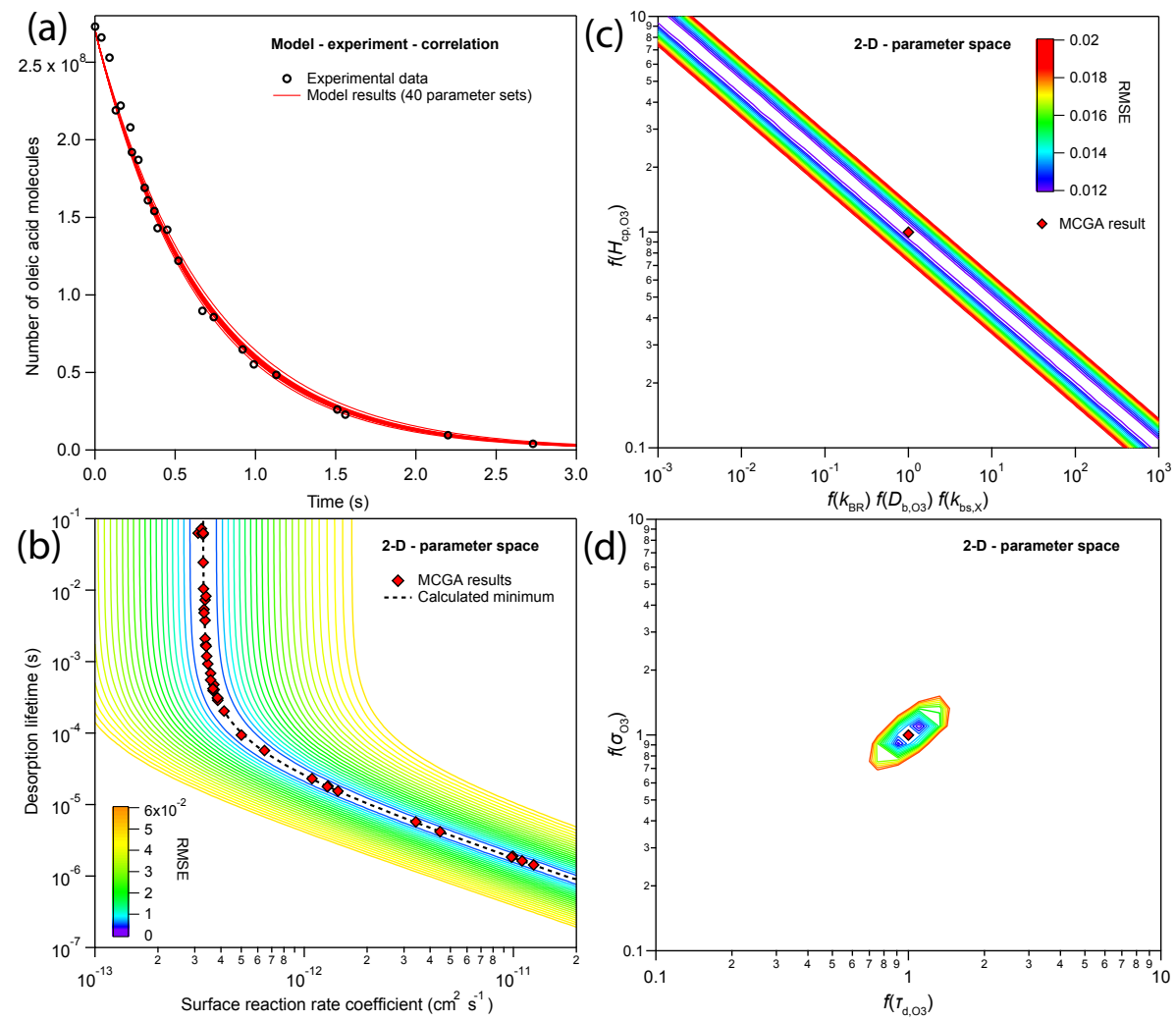

Figure 3. (a) Results from repeatedly fitting a kinetic model to a single experimental decay curve (adopted from Hearn et al., 2005). MCGA was used to optimize two model parameters, a surface reaction rate coefficient and the desorption lifetime of the gas-phase oxidant. All other model parameters remained fixed. (b) Visualization of the MCGA findings on the two-dimensional optimization hypersurface. The hypersurface (contour lines represent the root mean square deviances) exhibits no unique minimum due to insufficiently broad experimental data and optimization result (red diamonds) scatter along the extended minimum (black dashed line). Panels (c) and (d) show exemplary optimization hypersurfaces with two parameters showing an elongated (c) or a distinct minimum (d). Panels (c) and (d) are reproduced from Berkemeier et al. (2016) with permission from the PCCP Owner Societies.

of the bulk reaction rate coefficient $\left(k_{\mathrm{BR}}\right)$ with the bulk diffusivity of ozone $\left(D_{\mathrm{b}, \mathrm{O}_{3}}\right)$ and the bulk-to-surface transport coefficient of ozone $\left(k_{\mathrm{bs}, \mathrm{O}_{3}}\right)$ are fully non-orthogonal. Figure $3 \mathrm{~d}$ shows a single, well-defined optimum parameter set for the effective molecular cross section of ozone $\left(\sigma_{\mathrm{O}_{3}}\right)$ and the desorption lifetime of ozone $\left(\tau_{\mathrm{d}, \mathrm{O}_{3}}\right)$, indicating that these parameters are fully orthogonal for the experimental data fit in that study.

The prerequisite of a successful optimization is to fit a sufficiently broad experimental data set so that a unique and accurate set of fitting parameters is obtained. Thus, both of the conditions above must be avoided. This may be achieved by including additional experimental data, especially from a different experimental technique or over a different timescale so that the system might sample another limiting behaviour. In the data given in Fig. 3 above, for example, measuring full time series at different oxidant concentrations may help to constrain the oxidant's desorption lifetime. However, if a model has too many free parameters (or especially parameters that are not well-constrained by experimental data), it may be necessary to reduce the model complexity or fix some of the parameters. We therefore recommend using data sets obtained from a range of different experimental techniques to ensure this variability if they are available, and using models with as few free parameters as possible.

In the example above, it was possible to use brute-force sampling to determine the true optimization hypersurface (contour lines) for comparison to the MCGA results. Of course, in typical applications, the number and range of input parameters makes such a search prohibitive. The computational feasibility of an optimization depends crucially on the size of the input parameter space, i.e. number and possible range of all parameters. Using an unreasonably large range for input parameters increases the possibility of finding nonphysical solutions that fit the experimental data. The input parameter space can be reduced based on a priori knowledge from laboratory experiments and theoretical calculations. Parameters can be narrowed down by laboratory experiments (e.g. bulk experiments for derivation of trace gas solubility), by physics (e.g. the upper limit of the accommodation coef- 
Table 1. Previous studies applying the MCGA.

\begin{tabular}{|c|c|}
\hline Study & Reaction system \\
\hline Berkemeier et al. (2013) & Oleic acid $+\mathrm{O}_{3}$ \\
\hline Arangio et al. (2015) & $\begin{array}{l}\text { Levoglucosan and } \\
\text { abietic acid }+\mathrm{OH}\end{array}$ \\
\hline Kampf et al. (2015) & Protein $+\mathrm{O}_{3}$ \\
\hline Hosny et al. (2016) & Oleic acid $+\mathrm{O}_{3}$ \\
\hline Berkemeier et al. (2016) & Shikimic acid $+\mathrm{O}_{3}$ \\
\hline Tong et al. (2016) & $\begin{array}{l}\text { OH formation by secondary or- } \\
\text { ganic aerosol } \\
\text { decomposition in water }\end{array}$ \\
\hline Lakey et al. (2016a) & $\begin{array}{l}\text { Reactive oxygen species and } \\
\mathrm{PM}_{2.5} \text { in lung lining fluid }\end{array}$ \\
\hline Lakey et al. (2016b) & Skin lipid (squalene) $+\mathrm{O}_{3}$ \\
\hline
\end{tabular}

ficient at unity), or by simulations (e.g. molecular dynamics simulations to estimate the surface accommodation coefficient and desorption lifetime as in Vieceli et al. (2005) and Julin et al. (2013)). Note that in the example given in Fig. 3b, the two parameters were not truly independent, so that constraining either model parameter from a priori information would constrain the other parameter. In multi-parameter optimizations, where many such dependencies might exist, this can lead to a significant reduction in solution space.

\section{Application of MCGA in atmospheric multiphase chemistry}

The MCGA has been previously applied to chemical reaction systems of atmospheric relevance (Table 1). The essential parameters we use to describe an atmospheric multiphase chemical kinetic system of reactive trace gases $X$ and bulk material $Y$ include chemical reaction rate coefficients at the surface $\left(k_{\mathrm{SLR}}\right)$ and in the bulk $\left(k_{\mathrm{BR}}\right)$ of aerosol particles; bulk diffusion coefficients of reactive trace gases $\left(D_{\mathrm{b}, X}\right)$ and the bulk matrix $\left(D_{\mathrm{b}, Y}\right)$; accommodation coefficients $\left(\alpha_{\mathrm{s}, X}\right)$ and desorption lifetimes $\left(\tau_{\mathrm{d}, X}\right)$ of trace gases to the particle surface to determine transient and equilibrium adsorption behaviour; and equilibrium constants for the solubility of reactive trace gases $\left(K_{\mathrm{sol}, \mathrm{cc}, X}\right)$, typically expressed in terms of Henry's law coefficients $\left(H_{\mathrm{cp}, X}\right)$ (Pöschl et al., 2007; Ammann and Pöschl, 2007; Shiraiwa et al., 2010; Berkemeier et al., 2013).

In its first application the MCGA was used to fit individual data sets of the decay of oleic acid upon ozonolysis (Berkemeier et al., 2013), highlighting the need for fitting to multiple experimental data sets to constrain kinetic parameters. This was done in further studies that investigated gas uptake to (semi-)solid organic material in coated-wall flow-tube reactors (Arangio et al., 2015; Berkemeier et al., 2016), ozoneinduced protein oligomerization in bulk solutions (Kampf et al., 2015), viscosity change upon alkene ozonolysis as mea- sured with fluorescence microscopy (Hosny et al., 2016), the redox-cycling reactions in the human lung lining fluid (Lakey et al., 2016a), and ozonolysis of squalene contained in human skin lipids (Lakey et al., 2016b). In each of these studies, a large set of model input parameters was optimized to several experimental data sets to constrain the input parameter space. In the following, we review results previously obtained by the MCGA to demonstrate its utility in determining kinetic parameters, assimilating large data sets, and detecting ill-defined problems.

In Berkemeier et al. (2016), 11 parameters, under 12 distinct experimental conditions and using a single set of kinetic parameters, were varied simultaneously to fit the ozone uptake to shikimic acid films over many hours (Fig. 4). The model was found to accurately describe the humidity- and concentration-dependence of ozone uptake and a high correlation between model output and experimental data was achieved. During optimization, a subset of six parameters, including diffusivity coefficients and trace gas solubility, was allowed to increase or decrease monotonically over six steps in relative humidity, resulting in a total of 41 optimized parameter values. Despite this large number of optimization parameters, a well-constrained parameter set could be obtained due to the large depth in training data and by applying a priori information.

In another study investigating the oxidation of biomass burning tracers with hydroxyl radicals (Arangio et al., 2015), repeated execution of MCGA revealed a remaining uncertainty in the kinetic parameters obtained from optimization to the two experimental data sets (Fig. 5). While some parameters could be narrowly constrained (diffusion coefficient of the organic matrix, $D_{\text {org }}$ ), others were subject to larger uncertainties (surface layer reaction rate constant, $k_{\mathrm{SLR}}$; desorption lifetime, $\left.\tau_{\mathrm{d}}\right)$. Note that while these parameters seem almost unconstrained in Fig. 5, this uncertainty is due to the presence of non-orthogonal parameter pairs. As detailed in Fig. 3 and in Arangio et al. (2015), only specific combinations of the non-orthogonal parameters will lead to agreement between model and experiment. This knowledge can be used to constrain these parameters in further experiments.

\section{Conclusions}

The MCGA addresses the problem of extracting physical and chemical parameters from experimental data. The algorithm allows the user to assimilate multiple data sets and its random sampling approach reduces the bias which may arise in more user-directed optimization methods. Unlike simple gradientbased optimization methods, MCGA can thus be used as a statistical tool that not only detects unconstrained parameters but also finds dependencies between unconstrained parameters. The results can be applied in process models and may serve to direct future experimental studies, e.g. to drive a reaction system into regimes in which the remaining uncon- 

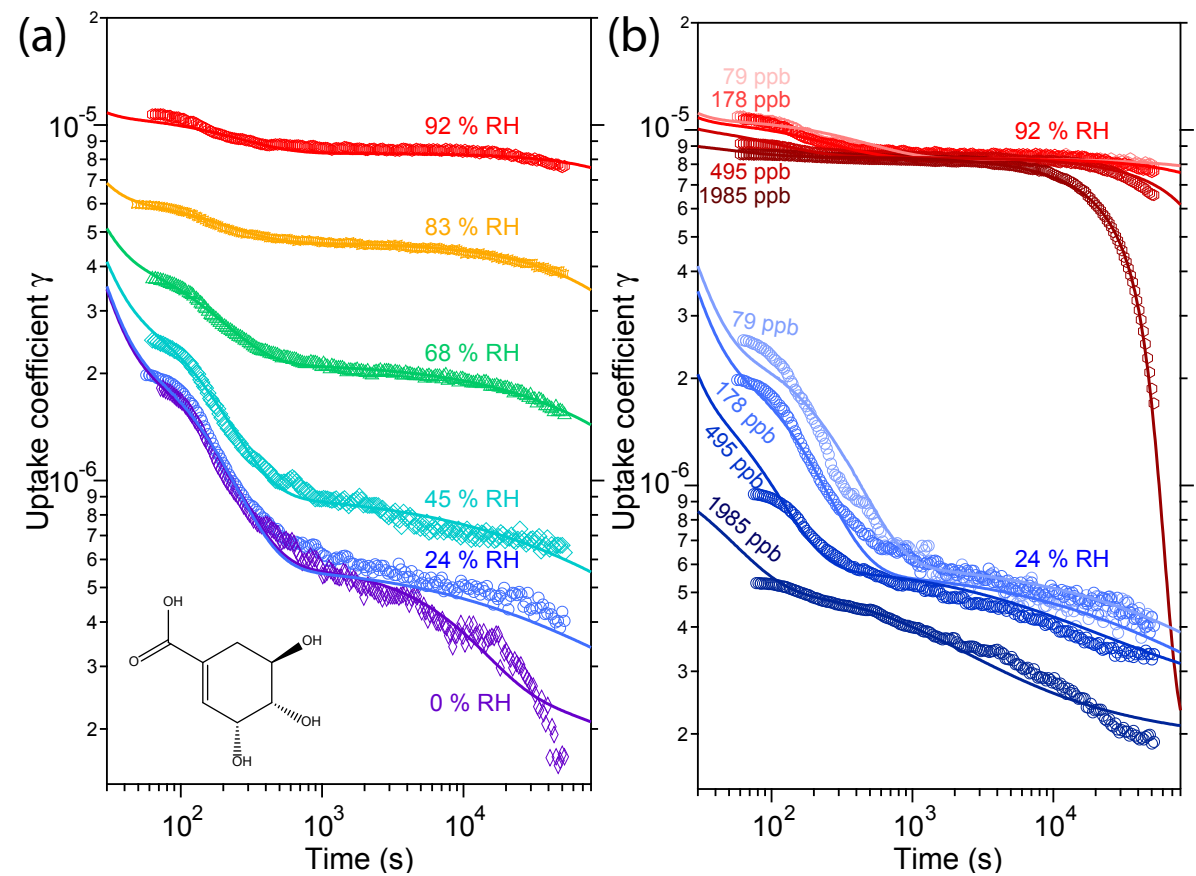

Figure 4. Observed (markers) and modelled (lines) uptake coefficients of ozone on a thin film of shikimic acid as a function of exposure time. (a) Uptake coefficients at 178 ppb ozone gas-phase concentration $\left[\mathrm{O}_{3}\right]_{\mathrm{g}}$ at different relative humidities of $0,24,45,68,83$, and $92 \%$. The structural formula of shikimic acid is displayed in the bottom left corner. (b) Uptake coefficients at $24 \%$ RH (blue solid lines) and $92 \%$ $\mathrm{RH}$ (red solid lines) with different $\left[\mathrm{O}_{3}\right]_{\mathrm{g}}$ of $79,178,495$, and $1985 \mathrm{ppb}$. Reproduced from Berkemeier et al. (2016) with permission from the PCCP Owner Societies.

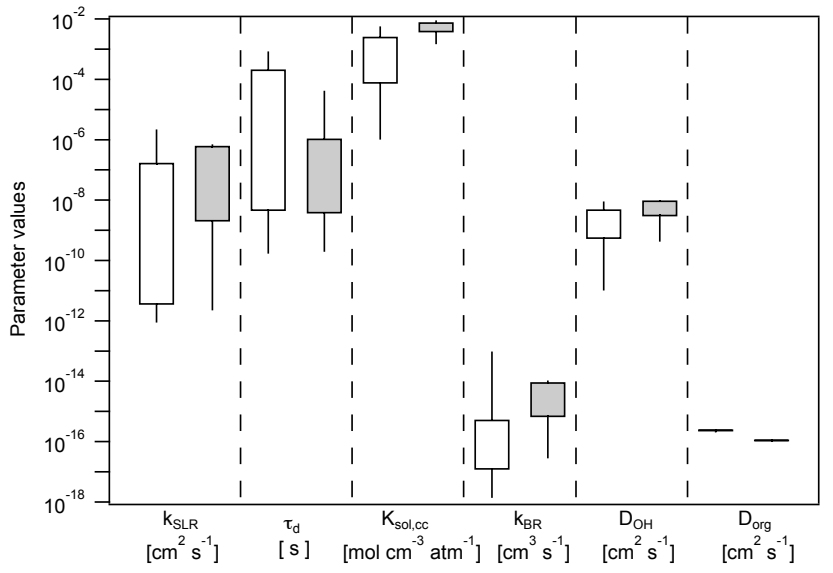

Figure 5. Kinetic parameters for multiphase chemical reactions of $\mathrm{OH}$ with levoglucosan (white) and abietic acid (grey) determined by the MCGA method of fitting the experimental data with the KM-GAP model. The ranges of parameters are depicted as a box-whisker plot (the percentiles of 10, 25, 75, and $90 \%$ are shown). Reprinted with permission from Arangio et al. (2015). Copyright 2015 American Chemical Society.

strained parameters have high sensitivity. MCGA could also be used to constrain chemical reaction systems in the postanalysis of field and laboratory studies: starting with a large set of model input parameters (i.e. chemical reactions, physical processes), data from various measurement campaigns could be combined, reconciled, and in a further step used to reduce the number of model input parameters to the key processes necessary to describe all measurement data. MCGA may be a powerful and useful tool to constrain kinetic parameters and reaction rate coefficients in models that study the formation of secondary organic aerosol in reaction chambers (Chan et al., 2007; Shiraiwa et al., 2013; Cappa et al., 2013; Riedel et al., 2016). It could be suitable for fine-tuning of reaction rates in large reaction mechanisms of atmospheric chemistry, such as the Master Chemical Mechanism (MCM; Jenkin et al., 1997; Saunders et al., 2003), the Gas-Aerosol Model for Mechanism Analysis (GAMMA; McNeill et al., 2012), or the Chemical Aqueous Phase Radical Mechanism (CAPRAM; Herrmann et al., 1999). Multiple experimental data sets from a broad range of techniques could be used with the algorithm to narrow down difficult-to-measure reaction rate coefficients, provide uncertainty estimates, and reconcile experiments across different research groups and facilities.

Data availability. No data sets were used in this article. 
Competing interests. The authors declare that they have no conflict of interest.

Disclaimer. Any opinions, findings, and conclusions or recommendations expressed in this material are those of the authors and do not necessarily reflect the views of the US National Science Foundation.

Acknowledgements. Thomas Berkemeier was supported by the Max Planck Graduate Center with the Johannes GutenbergUniversität Mainz (MPGC). Andrew J. Huisman was supported by the United States National Science Foundation under award no. IRFP 1006117 and by ETH Zürich. We gratefully acknowledge Geoffrey D. Smith for providing published data in tabulated form. The authors would like to thank Andrea Pozzer, Christian Pfrang, and Claudia Marcolli for stimulating discussions and support and John Rieffel for his expertise in algorithms.

The article processing charges for this open-access publication were covered by the Max Planck Society.

Edited by: David Topping

Reviewed by: three anonymous referees

\section{References}

Abbatt, J. P. D., Lee, A. K. Y., and Thornton, J. A.: Quantifying trace gas uptake to tropospheric aerosol: recent advances and remaining challenges, Chem. Soc. Rev., 41, 6555-6581, 2012.

Ammann, M. and Pöschl, U.: Kinetic model framework for aerosol and cloud surface chemistry and gas-particle interactions - Part 2: Exemplary practical applications and numerical simulations, Atmos. Chem. Phys., 7, 6025-6045, https://doi.org/10.5194/acp7-6025-2007, 2007.

Arangio, A. M., Slade, J. H., Berkemeier, T., Pöschl, U., Knopf, D. A., and Shiraiwa, M.: Multiphase Chemical Kinetics of OH Radical Uptake by Molecular Organic Markers of Biomass Burning Aerosols: Humidity and Temperature Dependence, Surface Reaction, and Bulk Diffusion, J. Phys. Chem. A, 119, 4533-4544, https://doi.org/10.1021/jp510489z, 2015.

Arora, J. S., Elwakeil, O. A., Chahande, A. I., and Hsieh, C. C.: Global optimization methods for engineering applications: A review, Struct. Optimization, 9, 137-159, https://doi.org/10.1007/bf01743964, 1995.

Berkemeier, T., Huisman, A. J., Ammann, M., Shiraiwa, M., Koop, T., and Pöschl, U.: Kinetic regimes and limiting cases of gas uptake and heterogeneous reactions in atmospheric aerosols and clouds: a general classification scheme, Atmos. Chem. Phys., 13, 6663-6686, https://doi.org/10.5194/acp-13-6663-2013, 2013.

Berkemeier, T., Steimer, S. S., Krieger, U. K., Peter, T., Poschl, U., Ammann, M., and Shiraiwa, M.: Ozone uptake on glassy, semi-solid and liquid organic matter and the role of reactive oxygen intermediates in atmospheric aerosol chemistry, Phys. Chem. Chem. Phys., 18, 12662-12674, https://doi.org/10.1039/C6CP00634E, 2016.

Cappa, C. D., Zhang, X., Loza, C. L., Craven, J. S., Yee, L. D., and Seinfeld, J. H.: Application of the Statistical Oxidation Model
(SOM) to Secondary Organic Aerosol formation from photooxidation of C12 alkanes, Atmos. Chem. Phys., 13, 1591-1606, https://doi.org/10.5194/acp-13-1591-2013, 2013.

Chan, A. W. H., Kroll, J. H., Ng, N. L., and Seinfeld, J. H.: Kinetic modeling of secondary organic aerosol formation: effects of particle- and gas-phase reactions of semivolatile products, Atmos. Chem. Phys., 7, 4135-4147, https://doi.org/10.5194/acp-74135-2007, 2007.

Finlayson-Pitts, B. J.: Reactions at surfaces in the atmosphere: integration of experiments and theory as necessary (but not necessarily sufficient) for predicting the physical chemistry of aerosols, Phys. Chem. Chem. Phys., 11, 7760-7779, https://doi.org/10.1039/b906540g, 2009.

George, I. J. and Abbatt, J. P. D.: Heterogeneous oxidation of atmospheric aerosol particles by gas-phase radicals, Nat. Chem., 2, 713-722, https://doi.org/10.1038/nchem.806, 2010.

Hanson, D. R., Ravishankara, A. R., and Solomon, S.: Heterogeneous reactions in sulfuric acid aerosols - A framework for model calculations, J. Geophys. Res.-Atmos., 99, 3615-3629, https://doi.org/10.1029/93jd02932, 1994.

Hearn, J. D., Lovett, A. J., and Smith, G. D.: Ozonolysis of oleic acid particles: evidence for a surface reaction and secondary reactions involving Criegee intermediates, Phys. Chem. Chem. Phys., 7, 501-511, https://doi.org/10.1039/b414472d, 2005.

Herrmann, H., Ervens, B., Nowacki, P., Wolke, R., and Zellner, R.: A chemical aqueous phase radical mechanism for tropospheric chemistry, Chemosphere, 38, 1223-1232, https://doi.org/10.1016/S0045-6535(98)00520-7, 1999.

Hosny, N. A., Fitzgerald, C., Vysniauskas, A., Athanasiadis, A., Berkemeier, T., Uygur, N., Pöschl, U., Shiraiwa, M., Kalberer, M., Pope, F. D., and Kuimova, M. K.: Direct imaging of changes in aerosol particle viscosity upon hydration and chemical aging, Chem. Sci., 7, 1357-1367, https://doi.org/10.1039/C5SC02959G, 2016.

Jenkin, M. E., Saunders, S. M., and Pilling, M. J.: The tropospheric degradation of volatile organic compounds: a protocol for mechanism development, Atmos. Environ., 31, 81-104, https://doi.org/10.1016/S1352-2310(96)00105-7, 1997.

Julin, J., Shiraiwa, M., Miles, R. E. H., Reid, J. P., Pöschl, U., and Riipinen, I.: Mass Accommodation of Water: Bridging the Gap Between Molecular Dynamics Simulations and Kinetic Condensation Models, J. Phys. Chem. A, 117, 410-420, https://doi.org/10.1021/jp310594e, 2013.

Kampf, C. J., Liu, F., Reinmuth-Selzle, K., Berkemeier, T., Meusel, H., Shiraiwa, M., and Pöschl, U.: Protein Cross-Linking and Oligomerization through Dityrosine Formation upon Exposure to Ozone, Environ. Sci. Technol., 49, 10859-10866, https://doi.org/10.1021/acs.est.5b02902, 2015.

Kolb, C. E., Cox, R. A., Abbatt, J. P. D., Ammann, M., Davis, E. J., Donaldson, D. J., Garrett, B. C., George, C., Griffiths, P. T., Hanson, D. R., Kulmala, M., McFiggans, G., Pöschl, U., Riipinen, I., Rossi, M. J., Rudich, Y., Wagner, P. E., Winkler, P. M., Worsnop, D. R., and O' Dowd, C. D.: An overview of current issues in the uptake of atmospheric trace gases by aerosols and clouds, Atmos. Chem. Phys., 10, 10561-10605, https://doi.org/10.5194/acp-1010561-2010, 2010.

Lakey, P. S. J., Berkemeier, T., Tong, H., Arangio, A. M., Lucas, K., Pöschl, U., and Shiraiwa, M.: Chemical exposureresponse relationship between air pollutants and reactive oxy- 
gen species in the human respiratory tract, Sci. Rep., 6, 32916, https://doi.org/10.1038/srep32916, 2016a.

Lakey, P. S. J., Wisthaler, A., Berkemeier, T., Mikoviny, T., Pöschl, U., and Shiraiwa, M.: Chemical kinetics of multi-phase reactions between ozone and human skin lipids: implications for indoor air quality and health effects, Indoor Air, 1-13, https://doi.org/10.1111/ina.12360, 2016b.

Lowe, S., Partridge, D. G., Topping, D., and Stier, P.: Inverse modelling of Köhler theory - Part 1: A response surface analysis of $\mathrm{CCN}$ spectra with respect to surfaceactive organic species, Atmos. Chem. Phys., 16, 10941-10963, https://doi.org/10.5194/acp-16-10941-2016, 2016.

McNeill, V. F., Woo, J. L., Kim, D. D., Schwier, A. N., Wannell, N. J., Sumner, A. J., and Barakat, J. M.: Aqueous-phase secondary organic aerosol and organosulfate formation in atmospheric aerosols: A modeling study, Environ. Sci. Technol., 46, 8075-8081, https://doi.org/10.1021/es3002986, 2012.

Partridge, D. G., Vrugt, J. A., Tunved, P., Ekman, A. M. L., Struthers, H., and Sorooshian, A.: Inverse modelling of cloud-aerosol interactions - Part 2: Sensitivity tests on liquid phase clouds using a Markov chain Monte Carlo based simulation approach, Atmos. Chem. Phys., 12, 2823-2847, https://doi.org/10.5194/acp-12-2823-2012, 2012.

Pöschl, U. and Shiraiwa, M.: Multiphase Chemistry at the Atmosphere-Biosphere Interface Influencing Climate and Public Health in the Anthropocene, Chem. Rev., 115, 4440-4475, https://doi.org/10.1021/cr500487s, 2015.

Pöschl, U., Rudich, Y., and Ammann, M.: Kinetic model framework for aerosol and cloud surface chemistry and gas-particle interactions - Part 1: General equations, parameters, and terminology, Atmos. Chem. Phys., 7, 5989-6023, https://doi.org/10.5194/acp7-5989-2007, 2007.

Riedel, T. P., Lin, Y.-H., Zhang, Z., Chu, K., Thornton, J. A., Vizuete, W., Gold, A., and Surratt, J. D.: Constraining condensed-phase formation kinetics of secondary organic aerosol components from isoprene epoxydiols, Atmos. Chem. Phys., 16, 1245-1254, https://doi.org/10.5194/acp-161245-2016, 2016.

Roldin, P., Eriksson, A. C., Nordin, E. Z., Hermansson, E., Mogensen, D., Rusanen, A., Boy, M., Swietlicki, E., Svenningsson, B., Zelenyuk, A., and Pagels, J.: Modelling non-equilibrium secondary organic aerosol formation and evaporation with the aerosol dynamics, gas- and particle-phase chemistry kinetic multilayer model ADCHAM, Atmos. Chem. Phys., 14, 7953-7993, https://doi.org/10.5194/acp-14-7953-2014, 2014.

Saunders, S. M., Jenkin, M. E., Derwent, R. G., and Pilling, M. J.: Protocol for the development of the Master Chemical Mechanism, MCM v3 (Part A): tropospheric degradation of nonaromatic volatile organic compounds, Atmos. Chem. Phys., 3, 161-180, https://doi.org/10.5194/acp-3-161-2003, 2003.
Shiraiwa, M., Pfrang, C., and Pöschl, U.: Kinetic multi-layer model of aerosol surface and bulk chemistry (KM-SUB): the influence of interfacial transport and bulk diffusion on the oxidation of oleic acid by ozone, Atmos. Chem. Phys., 10, 3673-3691, https://doi.org/10.5194/acp-10-3673-2010, 2010.

Shiraiwa, M., Pfrang, C., Koop, T., and Pöschl, U.: Kinetic multilayer model of gas-particle interactions in aerosols and clouds (KM-GAP): linking condensation, evaporation and chemical reactions of organics, oxidants and water, Atmos. Chem. Phys., 12, 2777-2794, https://doi.org/10.5194/acp-12-2777-2012, 2012.

Shiraiwa, M., Yee, L. D., Schilling, K. A., Loza, C. L., Craven, J. S., Zuend, A., Ziemann, P. J., and Seinfeld, J. H.: Size distribution dynamics reveal particle-phase chemistry in organic aerosol formation, P. Natl. Acad. Sci. USA, 110, 11746-11750, https://doi.org/10.1073/pnas.1307501110, 2013.

Shiraiwa, M., Berkemeier, T., Schilling-Fahnestock, K. A., Seinfeld, J. H., and Pöschl, U.: Molecular corridors and kinetic regimes in the multiphase chemical evolution of secondary organic aerosol, Atmos. Chem. Phys., 14, 8323-8341, https://doi.org/10.5194/acp-14-8323-2014, 2014.

Smith, G. D., Woods, E., Baer, T., and Miller, R. E.: Aerosol uptake described by numerical solution of the diffusion - Reaction equations in the particle, J. Phys. Chem. A, 107, 9582-9587, https://doi.org/10.1021/jp021843a, 2003.

Steimer, S. S., Berkemeier, T., Gilgen, A., Krieger, U. K., Peter, T., Shiraiwa, M., and Ammann, M.: Shikimic acid ozonolysis kinetics of the transition from liquid aqueous solution to highly viscous glass, Phys. Chem. Chem. Phys., 17, 3110131109, https://doi.org/10.1039/C5CP04544D, 2015.

Vieceli, J., Roeselova, M., Potter, N., Dang, L. X., Garrett, B. C., and Tobias, D. J.: Molecular dynamics simulations of atmospheric oxidants at the air-water interface: Solvation and accommodation of $\mathrm{OH}$ and $\mathrm{O}_{3}$, J. Phys. Chem. B, 109, 15876-15892, https://doi.org/10.1021/jp051361+, 2005.

Worsnop, D. R., Morris, J. W., Shi, Q., Davidovits, P., and Kolb, C. E.: A chemical kinetic model for reactive transformations of aerosol particles, Geophys. Res. Lett., 29, 57-1-57-4, https://doi.org/10.1029/2002gl015542, 2002.

Zobrist, B., Soonsin, V., Luo, B. P., Krieger, U. K., Marcolli, C., Peter, T., and Koop, T.: Ultra-slow water diffusion in aqueous sucrose glasses, Phys. Chem. Chem. Phys., 13, 3514-3526, https://doi.org/10.1039/c0cp01273d, 2011.

Zuend, A. and Seinfeld, J. H.: A practical method for the calculation of liquid-liquid equilibria in multicomponent organicwater-electrolyte systems using physicochemical constraints, Fluid Phase Equilibr., 337, 201-213, 2013. 